\title{
SIMPLICITY OF TWISTED C*-ALGEBRAS OF TOPOLOGICAL HIGHER-RANK GRAPHS
}

\author{
BECKY ARMSTRONG
}

(Received 4 February 2020; first published online 13 March 2020)

2010 Mathematics subject classification: primary 46L05.

Keywords and phrases: $\mathrm{C}^{*}$-algebra, simplicity, topological higher-rank graph, groupoid.

In a recent series of papers, Kumjian, Pask and Sims [2-5] have investigated the effect of 'twisting' $\mathrm{C}^{*}$-algebras associated to higher-rank graphs using a categorical 2 -cocycle on the graph. This work has included a characterisation of simplicity for these twisted $\mathrm{C}^{*}$-algebras in terms of the underlying graphical and cohomological data. In this thesis, we initiate the study of twisted $\mathrm{C}^{*}$-algebras associated to topological higher-rank graphs using groupoid techniques and we characterise simplicity of these $\mathrm{C}^{*}$-algebras.

For each cofinal, proper, source-free topological higher-rank graph, and each continuous 2-cocycle on the associated boundary-path groupoid, we consider the twisted groupoid $\mathrm{C}^{*}$-algebra in the sense of Renault. We show that the quotient of the boundary-path groupoid by the interior of its isotropy subgroupoid acts on the Cartesian product of the infinite-path space of the graph and the dual group of a particular subgroup of the periodicity group of the graph that is dependent on the cohomological data. We refer to this action as the spectral action.

To prove our simplicity characterisation, we first extend results of Brown, Nagy, Reznikoff, Sims and Williams [1] to characterise injectivity of homomorphisms of the reduced twisted $\mathrm{C}^{*}$-algebra associated to any Hausdorff étale groupoid $\mathcal{G}$ and continuous 2-cocycle on $\mathcal{G}$ in terms of injectivity of homomorphisms of the reduced twisted $\mathrm{C}^{*}$-algebra associated to the interior of the isotropy of $\mathcal{G}$. We apply this result to prove that a twisted $C^{*}$-algebra of a topological higher-rank graph is simple if the associated spectral action is minimal. We complete the proof of our characterisation by assuming that the spectral action is not minimal and constructing a nonzero representation of the twisted $\mathrm{C}^{*}$-algebra with nontrivial kernel. Our characterisation of simplicity generalises the analogous result of Kumjian, Pask and Sims pertaining to twisted $\mathrm{C}^{*}$-algebras of (discrete) higher-rank graphs.

Thesis submitted to the University of Sydney in June 2019; degree approved on 23 August 2019; supervisors Nathan Brownlowe and Aidan Sims.

(C) 2020 Australian Mathematical Publishing Association Inc. 


\section{References}

[1] J. H. Brown, G. Nagy, S. Reznikoff and D. P. Williams, 'Cartan subalgebras in $C^{*}$-algebras of Hausdorff étale groupoids', Integral Equations Operator Theory 85(1) (2016), 109-126.

[2] A. Kumjian, D. Pask and A. Sims, 'Homology for higher-rank graphs and twisted $C^{*}$-algebras', J. Funct. Anal. 263(6) (2012), 1539-1574.

[3] A. Kumjian, D. Pask and A. Sims, 'On the $K$-theory of twisted higher-rank graph $C^{*}$-algebras', J. Math. Anal. Appl. 401(1) (2013), 104-113.

[4] A. Kumjian, D. Pask and A. Sims, 'On twisted higher-rank graph $C^{*}$-algebras', Trans. Amer. Math. Soc. 367(7) (2015), 5177-5216.

[5] A. Kumjian, D. Pask and A. Sims, 'Simplicity of twisted $C^{*}$-algebras of higher-rank graphs and crossed products by quasifree actions', J. Noncommut. Geom. 10(2) (2016), 515-549.

BECKY ARMSTRONG, School of Mathematics and Statistics, University of Sydney, Camperdown, NSW 2006, Australia

e-mail: becky.armstrong@sydney.edu.au 